\title{
The genus Nipponodrasterius Kishii (Coleoptera, Elateridae, Agrypninae), a junior synonym of the genus Gamepenthes Fleutiaux (Coleoptera, Elateridae, Elaterinae), with review of the Japanese Gamepenthes species
}

\author{
Kôichi Arimoto', Hisayuki Arimoto \\ I Laboratory of Animal Ecology, Graduate School of Science, Kyoto University, Kyoto, 606-8502 Japan \\ 2 Tedukayama-nishi, Osaka, 558-0052 Japan \\ Corresponding author: Kôichi Arimoto (elateridbeetle@gmail.com)
}

Academic editor: H. Douglas | Received 6 July 2020 | Accepted 21 October 2020 | Published 16 December 2020

http://zoobank.org/DBBDD33A-34E6-42F1-AAEB-5FAD41349065

Citation: Arimoto K, Arimoto H (2020) The genus Nipponodrasterius Kishii (Coleoptera, Elateridae, Agrypninae), a junior synonym of the genus Gamepenthes Fleutiaux (Coleoptera, Elateridae, Elaterinae), with review of the Japanese Gamepenthes species. ZooKeys 1004: 109-127. https://doi.org/10.3897/zookeys.1004.56201

\begin{abstract}
The genus Nipponodrasterius Kishii, 1966 was established as a member of the subfamily Conoderinae Fleutiaux, 1919 (now tribe Oophorini Gistel, 1848; subfamily Agrypninae Candèze, 1857) based on N. alpicola. The genus was suggested to be unlikely to belong to Agrypninae because it lacks diagnostic features of the Agrypninae. However, there are no taxonomic treatments for the genus or species. Here, we review the status of the genus and species by examining the holotype of $N$. alpicola. Consequently, the genus was found to be a junior synonym of the genus Gamepenthes Fleutiaux, 1928 and N. alpicola was found to be a junior synonym of G. pictipennis (Lewis, 1894). We review all species of Gamepenthes in Japan and provide a key to species.
\end{abstract}

\section{Keywords}

Japan, Megapenthini, new synonym, Oophorini, Palearctic region, taxonomic review

Copyright K. Arimoto, H. Arimoto This is an open access article distributed under the terms of the Creative Commons Attribution License (CC BY 4.0), which permits unrestricted use, distribution, and reproduction in any medium, provided the original author and source are credited. 


\section{Introduction}

The monotypic elaterid genus Nipponodrasterius Kishii, 1966 was established as a member of the subfamily Conoderinae Fleutiaux, 1919 (currently tribe Oophorini Gistel, 1848; subfamily Agrypninae Candèze, 1857) based on Nipponodrasterius alpicola Kishii, 1966. This species is known only from the holotype collected on a mountain (altitude 2800 meters) in central Honshu, Japan. Its sex was not determined in the original description. The original description separates the genus from known members of tribe Oophorini using the following characteristics: rather flat body, antennomeres II and III small, antennomeres IV-X clearly serrate, elytral surface clearly granulated, and tarsomere IV shortly bilobed. Kishii (1987) redescribed this genus, modifying the original description slightly. He changed the description "tarsomere IV shortly bilobed" in Kishii (1966) to "tarsomere IV slightly enlarged apically". Moreover, he added an important character state to the generic description, claws without basal setae. Ôhira (1994) focused on the status of the claws and suggested that this genus is unlikely to belong to Agrypninae, because the presence of basal setae on the claws is a diagnostic feature of members of Agrypninae, absent only in genus Danosoma Thomson, 1859 (Hayek 1973). Ôhira (1994) did not examine specimens of the genus directly and did not revise the status of the genus.

We observed that N. alpicola is similar to species of the genus Gamepenthes Fleutiaux, 1928 in the tribe Megapenthini Gurjeva, 1973, subfamily Elaterinae Leach, 1815 based on these diagnostic features and a drawing of the dorsal habitus in the original description. In considering the systematic position of $N$. alpicola, the direction of the mouth-parts and the structure of the head capsule, procoxal cavity, prosternal process, mesocoxal cavity and mesosternum are also important characters (Ôhira 1970a; Stibick 1979; Schimmel 2003), however, these features were not described in Kishii $(1966,1987)$. The holotype of the type species required re-examination.

Five Gamepenthes species are distributed in Japan: G. ornatus (Lewis, 1894); G. pictipennis (Lewis, 1894); G. similis (Lewis, 1894); G. versipellis (Lewis, 1894); G. yoshidai Ôhira, 1995. Ôhira (1995a) reviewed the four Japanese species, except for G. yoshidai, and showed that they are clearly separated from each other by their aedeagi. Ôhira (1995a) provided a key to distinguish them, based mainly on body color, although members of the genus often show color variation. Subsequently, Ôhira (1995b) described a new species as G. yoshidai without showing its aedeagus. Hence, we examine the holotype of $N$. alpicola and review the Japanese Gamepenthes species including $G$. yoshidai in order to resolve the problem of Nipponodrasterius. Additionally, we provide a new key to these species based on external morphology.

\section{Material and methods}

We examined the holotype of $N$. alpicola and non-type specimens of the five Japanese Gamepenthes species, as well as the only paratype of G. yoshidai. The type specimens are in the collection of the Osaka Museum of Natural History (OMNH; Osaka, Japan). 
The non-type specimens are in the personal collection of the authors (Osaka, Japan) and will be donated to the $\mathrm{OMNH}$ in the future. Unique identifier numbers of the non-type specimens are: GO001-GO014; GP001-GP012; GS001-GS010; GV001-GV017; GY001, GY002. We found a slide of the male genitalia of the holotype of $N$. alpicola in OMNH (Fig. 5L), although its sex and genitalia were not mentioned in the original description. On the slide, the aedeagus of the holotype, missing the phallobase, had been mounted in balsam and distorted due to pressure exerted by the cover slip (Fig. 5J).

The methods used for observing and dissecting specimens, taking photographs, creating line drawings, and depositing the dissected parts follow Arimoto and Suzuki (2020).

The classification of the family Elateridae follows Kundrata et al. (2019), Bouchard et al. (2011), and Cate (2007). The morphological nomenclature system follows Costa et al. (2010) and Douglas (2011). The diagnosis of Gamepenthes was based on information from Ôhira (1970a, 1995a), Schimmel (2003), and evidence found during this study. The non-type specimens were identified using descriptions and figures in Lewis (1894) and Ôhira (1995a, b).

\section{Measurements and indices}

Measurements and indices were made following Arimoto and Suzuki (2020). Measurements are shown in millimeters. The following abbreviations are used:

$\begin{array}{ll}\text { BL } & \text { Body length from head to elytral apices } \\ \text { BW } & \text { Maximum body width } \\ \text { MAE } & \text { Maximum distance across the eyes } \\ \text { MBE } & \text { Minimum distance between the eyes } \\ \text { OI } & \text { Ocular index: MAE/MBE } \times 100 \\ \text { PL } & \text { Maximum pronotum length including hind angles } \\ \text { PML } & \text { Length of the midline of pronotum } \\ \text { PAW } & \text { Minimum pronotum width between anterior angles } \\ \text { PW } & \text { Maximum pronotum width including hind angles } \\ \text { PI } & \text { Pronotum index: PL/PW } \times 100 \\ \text { PWI } & \text { Pronotum width index: PAW/PW } \times 100 \\ \text { EL } & \text { Maximum elytra length } \\ \text { EW } & \text { Maximum elytra width } \\ \text { EI } & \text { Elytra index: EL/EW } \times 100 \\ \text { BI } & \text { Body index: EL/PL } \times 100\end{array}$

\section{Taxonomy}

\section{Genus Gamepenthes Fleutiaux, 1928}

Gamepenthes Fleutiaux, 1928: 158 (original description; type species: Megapenthes octomaculatus Schwarz, 1898; by original designation). 
Nipponodrasterius Kishii, 1966: 9 (original description; type species: Nipponodrasterius alpicola Kishii, 1966; by original designation); Kishii 1987: 62 (redescription). syn. nov.

Diagnosis. Head capsule oval in lateral view. Supra-antennal carina complete, rounded, depressed medially. Frontoclypeal region narrowed medially. Mouth-parts inferior. Antennae serrate from antennomere IV, without median longitudinal carina; antennomere IV longer than II-III combined in many, shorter than II-III combined in a few. Pronotum with median basal furrow, without sublateral incision near hind angles; hind angles unicarinate. Pronotosternal sutures not grooved or very shallowly grooved in front. Posterior edge of hypomeron straight mesally and then broadly rounded. Procoxal cavity partly closed behind by mesal projection of hypomeron. Prosternal process concave between procoxae, with subapical tooth in lateral view. Side of scutellum parallel on anterior half. Mesocoxal cavity open to mesepimeron and mesepisternum. Mesosternum separated by suture from metasternum. Outer edge of metacoxal plates wide in most, but narrowed in a few. Elytral surface with rasp-like punctures; elytral apical edge serrate and with small spines, serration very slight in some and then almost rounded. Tarsi simple; claws simple, without basal setae.

Ôhira (1970a, 1995a) stated that the apical elytral edge is more or less truncate; however, this is based on a misunderstanding.

Distribution. Oriental region (Bhutan, China, India, Indonesia, Laos, Malaysia, Myanmar, Nepal, Philippines, Taiwan, Thailand, Vietnam): 30 species (Schimmel 2003, 2004, 2006; Schimmel and Tarnawski 2009). Palearctic region (Japan, Russia): five species (Kishii 1999; Prosvirov 2013).

Ecology. Adults of the genus are often observed visiting flowers during the daytime. In Japan, Gamepenthes has been recorded visiting the flowers of Sambucus sp. and Viburnum sp. (family Adoxaceae), Angelica sp. (Apiaceae), Aralia cordata Thunb. (Araliaceae), Clethra barbinervis Siebold et Zucc. (Clethraceae), Neoshirakia japaonica (Siebold et Zucc.) Esser (Euphorbiaceae), Castanea crenata Siebold et Zucc. (Fagaceae), Hydrangea paniculata Siebold and H. serrata (Thunb.) Ser. var. acuminata (Siebold et Zucc.) Nakai (Hydrangeaceae), and Cimicifuga sp. (Ranunculaceae) (Ôhira, 1995a). This study adds flowers of Tilia japonica (Miq.) Simonk. (Malvaceae) to the records.

While most adult Gamepenthes individuals are not attracted to lights at night, $G$. similis has been collected by light traps (Ootsuka et al., 1981). We examined a few specimens of $G$. ornatus and $G$. pictipennis collected using simple light traps made with weak fluorescent lights.

Included species from Japan. Five species: G. ornatus (Lewis, 1894); G. pictipennis (Lewis, 1894); G. similis (Lewis, 1894); G. versipellis (Lewis, 1894); G. yoshidai Ôhira, 1995.

\section{Key to species of the genus Gamepenthes from Japan}

$1 \quad$ Body size large (BL: over $5.1 \mathrm{~mm}$ ) (Fig. 1A, B, D, E); antennomere II shorter than wide in male (Fig. 3A, C), almost as long as wide or slightly longer than 
wide in female (Fig. 3B, D); antennomere IV longer than II-III combined (Fig. 3A-D) 2

- $\quad$ Body size small (BL: under $5.0 \mathrm{~mm}$ ) (Fig. 1C); antennomere II longer than wide (Fig. 3E, F); antennomere IV shorter than II-III combined (Fig. 3E, F) G. similis (Lewis, 1894) Antennomere IV robust (1.36-1.72 times longer than wide in male, 1.661.79 times longer than wide in female) (Fig. 3A, B); lateral half of metacoxal plates not narrowed laterad, with sides wide (Fig. 3G); apical edge of elytra serrate (Fig. 3I) 3

- $\quad$ Antennomere IV elongate (1.74-2.06 times longer than wide in male, 1.962.17 times longer than wide in female) (Fig. 3C, D); lateral half of metacoxal plates narrowed laterad, with sides narrow (Fig. $3 \mathrm{H}$ ); apical edge of elytra rounded, without spines (Fig. 3J)

3 Body size smaller (BL: under $6.5 \mathrm{~mm}$ in male, under $7.1 \mathrm{~mm}$ in female) (Fig. 1A); pronotum moderately widened posterad (PWI: 150-156 in male, 161-174 in female) (Fig. 4A, B)

G. ornatus (Lewis, 1894)

- $\quad$ Body size larger (BL: over $6.5 \mathrm{~mm}$ in male, over $7.0 \mathrm{~mm}$ in female) (Fig. 1D); pronotum strongly widened posterad (PWI: 172-184 in male, 176-191 in female) (Fig. 4C, D)

G. versipellis (Lewis, 1894) Body robust (EI: 222-244, BI: 209-247) (Fig. 1B); prosternal process weakly inclined dorsad $\left(20-25^{\circ}\right)$ (Fig. 4E) G. pictipennis (Lewis, 1894) Body slender (EI: 259-265, BI: 254-282) (Fig. 1E); prosternal process strongly inclined dorsad (43-50 ) (Fig. 4F).

G. yoshidai Ôhira, 1995

\section{Gamepenthes ornatus (Lewis, 1894)}

Figures 1A, 3A, B, 4A, B, 7A

Megapenthes ornatus Lewis, 1894: 47 (original description; type locality: Yuyama, Kumamoto Prefecture, Kyushu, Japan).

Gamepenthes ornatus (Lewis, 1894); Kishii 1959: 58 (changed generic status).

Megapenthes ornatus aberrant form basalis Nakane, 1958: 87 (original description; type locality: Mt. Osore-zan, Aomori Prefecture, Honshu, Japan).

Material examined. 2 females, Japan, Honshu, Nara Prefecture, Yoshino District, Kamikitayama Village, Amagase, 11 VII 2004, Hisayuki Arimoto leg. [females: GO001, GO002]; 1 male, Japan, Honshu, Nara Prefecture, Yoshino District, Totsukawa Village, Asahi, Mt. Syakaga-take, 1450 m, 4 VIII 2019, Hisayuki Arimoto leg., from flowers of Tilia japonica [male:GO003]; 3 males, 1 female, Japan, Honshu, Wakayama Prefecture, Tanabe City, Mt. Gomadan-zan, 18 VII 2019, Hisayuki Arimoto leg. [males: GO004-GO006; female: GO007]; 2 females, Japan, Honshu, Nara Prefecture, Tenkawa Village, near Gyôjagaeshi Tunnel, 900 m, 16 VII 2017, Hisayuki Arimoto leg. [GO008, GO009]; 1 male, Japan, Honshu, Wakayama Prefecture, Tanabe City, Mt. Jyôgamori-yama, 18 VII 2019, Hisayuki Arimoto leg. [GO010]; 1 female, 

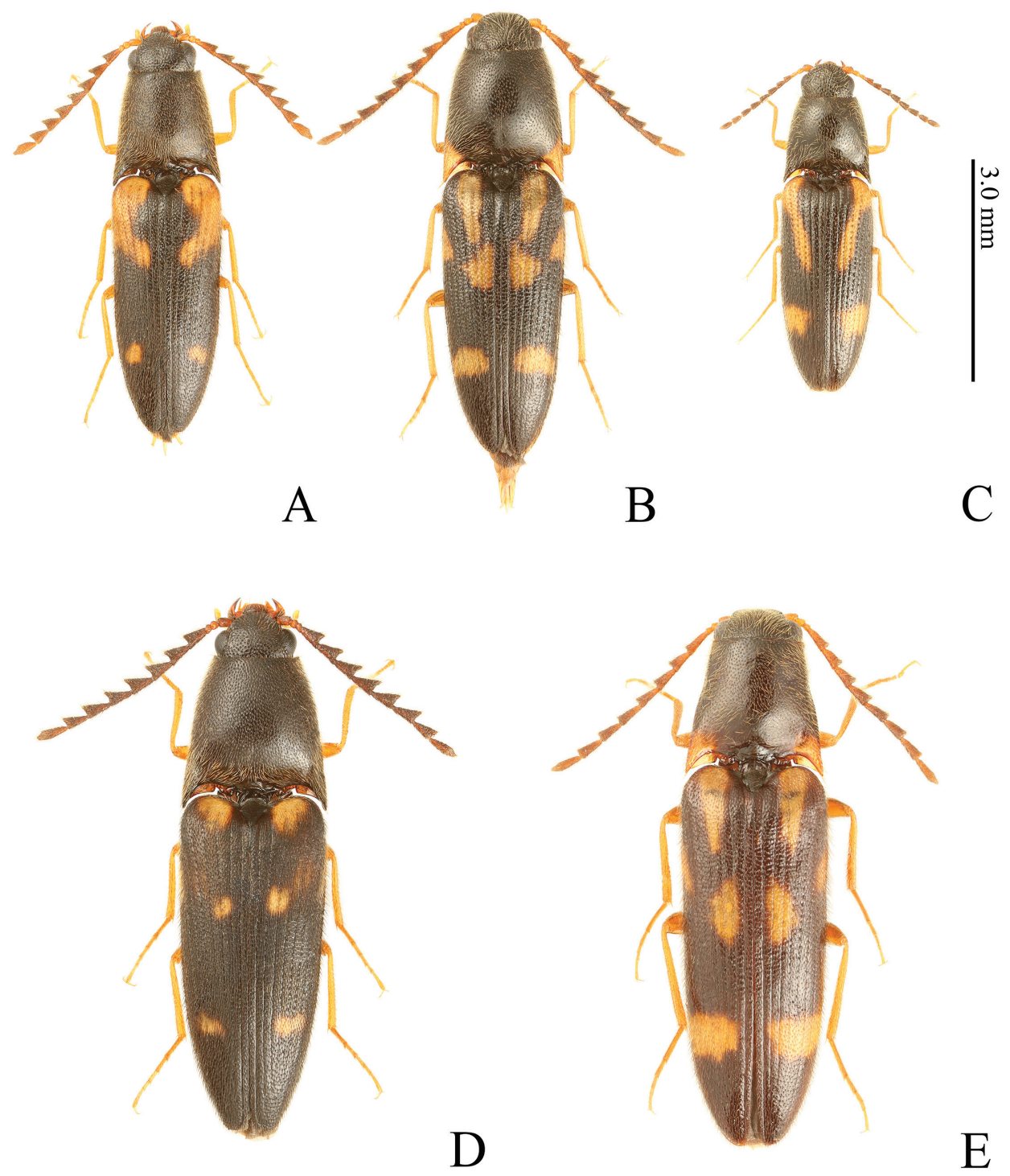

Figure I. Gamepenthes spp., male, habitus, dorsal view A G. ornatus [GO003] B G. pictipennis [GP010] C G. similis [GS003] D G. versipellis [GV006] E G. yoshidai [GY001].

Japan, Honshu, Okayama Prefecture, Tomada District, Kagamino Town, Neji, 8-15 VII 2016, Akihiko Watanabe leg., by light FIT [GO011]; 1 male, Japan, Kyushu, Kumamoto Prefecture, Uki City, Mt. Shiraiwa-yama, 16-17 VII 2016, Ryô Noda leg. [GO012]; 2 females, Japan, Kyushu, Miyazaki Prefecture, Nishiusuki District, Gokase Town, Mt. Shiraiwa-yama, 1347 m, 32³4'13.7"N, 13106'51.6"E, 30 VII 2011, Kôichi Arimoto leg. [GO013, GO014].

Diagnosis. Body robust (EI: 238-254, BI: 220-236). Prothorax black in male, brown in female. Each elytron with pale orange U-shaped marking basally. Antenno- 
mere II shorter than wide in male, almost as long as wide in female; III shorter than wide in male, longer than wide in female; IV robust (1.36-1.53 times longer than wide in male, 1.69-1.79 times longer than wide in female), longer than II-III combined (1.53-1.99 times as long as II-III combined in male, 1.02-1.10 times as long as in female). Prosternal process weakly inclined dorsad (24-38 $)$. Metacoxal plates not narrowed outwards on lateral half; outer edge wide. Apical edge of elytra serrate. Apex of parameres beyond pre-apical expansions equilateral triangular (apex length 0.8-0.9 times width of parameres at expansion).

Measurements. Male (6 spec.). BL: 5.29-6.18; BW: 1.41-1.72; MAE: 0.8931.07; MBE: 0.599-0.719; OI: 148-154; PL: 1.47-1.82; PML: 1.24-1.51; PAW: 0.925-1.08; PW: 1.41-1.70; PI: 105-107; PWI: 150-156; EL: 3.47-4.12; EW: 1.41-1.72; EI: 238-246; BI: 226-236. Female (8 spec.). BL: 5.86-7.03; BW: 1.571.93; MAE: 0.929-1.12; MBE: 0.620-0.764; OI: 141-150; PL: 1.71-2.12; PML: 1.42-1.81; PAW: 0.956-1.14; PW: 1.57-1.93; PI: 106-111; PWI: 161-174; EL: 3.90-4.67; EW: 1.57-1.93; EI: 242-254; BI: 220-230.

Distribution. Japan: Honshu, Shikoku, Kyushu.

Comparative notes. Gamepenthes ornatus and G. versipellis (Lewis, 1894) are often found sympatrically and are remarkably similar. They are distinguished by apical expansion of parameres beyond apical-lateral hooks (G. ornatus, apex length 0.8-0.9 times width of parameres at expansion; G. versipellis, $0.5-0.6$ times width of parameres at expansion) (Fig. 7A, D).

Ôhira (1995a) distinguished G. ornatus from G. versipellis in the key by the antennomere II ( $G$. ornatus, longer than wide; $G$. versipellis, shorter than wide) and color of the basal outer edge of the elytra (G. ornatus, pale orange, Fig. 1A; G. versipellis, black, Fig. 1D). However, in both species, the males' antennomere II is shorter than wide (Fig. 3A), and in females, it is almost as long as or slightly longer than wide (Fig. 3B). Moreover, their elytral base is variable in color, and we found specimens [GO001, GO016] of $G$. versipellis with a pale orange basal outer edge of the elytra. The two species are difficult to distinguish using antennomere II and elytral color. In females, pronotum coloration is a good diagnostic character ( $G$. ornatus, brown, Fig. 4B; $G$. versipellis, red, Fig. 4D) because no pronotum color variation has been found in either species. Considering the possibility that females with non-red pronota have been found, pronotum shape is the best diagnostic character for both sexes of the two species except for aedeagus (G. ornatus, PWI 150-156 in male and 161-174 in female; G. versipellis, PWI 172-184 in male and 176-191 in female) (Fig. 4A-D).

\section{Gamepenthes pictipennis (Lewis, 1894)}

Figures 1B, 2A-D, 3C, D, 4E, 5, 6, 7B

Melanoxanthus pictipennis Lewis, 1894: 48 (original description; type locality: Fukushima [Kisofukushima in Nagano Prefecture] and Nataksugawa [Nakatsugawa in Gifu Prefecture], Honshu, Japan).

Gamepenthes pictipennis (Lewis, 1894); Kishii 1959: 58 (changed generic status). 
Gamepenthes pictipennis aberrant form mizunoi Kishii, 1968: 14 (original description; type locality: Tokugô-tôge Pass, Nagano Prefecture, Honshu, Japan).

Nipponodrasterius alpicola Kishii, 1966: 9 (original description; type locality: Sampuku-tôge Pass, Kashio, Ôshika Village, Shimoina District, Nagano Prefecture, Honshu, Japan). syn. nov.

Material examined. Holotype of Nipponodrasterius alpicola. Male, Japan, Honshu, Nagano Prefecture, Shimoina District, Ôshika Village, Kashio, Sampuku-tôge Pass, 17-20, VII, 1956, H. Nitta leg. Verbatim label data (Fig. 5J): "Nipponodrasterius / alpicola / Kishii. 1966 / DET. T. KISHII. "66"; "HOLOTYPE"; "Sampuku pass / South Alps, Japan / July 17 20, 1956 / Coll. Nitta”; "2489”.

Non-type material. 1 male, Japan, Hokkaido, Uryû District, Horokanai Town, Syumarinai, 31 VII 1982, Koji Hosokawa leg. [GP001]; 1 female, Japan, Honshu, Gunma Prefecture, Ôsawa, 19 VII 1975, M. Yagi leg. [GP002]; 1 male, Japan, Honshu, Yamanashi Prefecture, Sudama Town, Masutomi spa, 9 VIII 1989, Yutaka Ishikawa leg. [GP003]; 1 female, same place as the former, 1300 m, 26 VII 1999, Yutaka Ishikawa leg. [GP004]; 1 female, same place as the former, ca $1300 \mathrm{~m}$, base of the western end of the Okuchichibu Mountains, 18 VII 1990, Yutaka Ishikawa leg. [GP005]; 1 male, Japan, Honshu, Yamanashi Prefecture, Kôshû City, Enzan, Hikawa Forest Road, 20 VIII 1989, K. Shindô leg. [GP006]; 1 male, Japan, Honshu, Gifu Prefecture, Takayama City, Shôkawa Town, Oganigô, Ôkuro Valley, 30 VI 2007, Hisayuki Arimoto leg. [GP007]; 1 female, Japan, Honshu, Gifu Prefecture, Ôno District, Shôkawa Village, Ogamigô, 12 VIII 1995, N. Yuzawa leg. [GP008]; 1 male, Japan, Honshu, Nara Prefecture, Tenkawa Village, Tsubonouchi Forest Road, 1200m, 21 VII 2008, Hisayuki Arimoto leg. [GP009]; 1 male, 1 female, Japan, Honshu, Okayama Prefecture, Tomada District, Kagamino Town, Neji, 8-15 VII 2016, Akihiko Watanabe leg., by light FIT [male: GP0010; female: GP011]; 1 male, Japan, Kyushu, Ôita Prefecture, Toyogoôno City, Ogata Town, Mt. Furusobo-san, 30 VII 2017, Yûji Tsutsumiuchi leg. [GP012].

Diagnosis. Body robust (EI: 222-244, BI: 209-247). Prothorax black; hind angles of pronotum yellow to orange in many, black in some. Each elytron with three separate yellow markings basally; basal markings connected in many, variably reduced in some. Antennomere II shorter than wide; antennomere III shorter than wide in male, slightly longer than wide in female; IV elongate (1.74-2.00 times longer than wide in male, 1.96-2.05 times longer than wide in female), longer than II-III combined (1.47-1.79 times as long as II-III combined in male, 1.21-1.40 times as long as II-III combined in female). Prosternal process weakly inclined dorsad $\left(20-25^{\circ}\right)$. Metacoxal plates narrowed outwards, with outer edge narrow. Apical edge of elytra rounded, without spines. Apex of parameres beyond pre-apical expansions widely triangular (apex length $0.1-0.2$ times width of parameres at expansion).

Measurements. Male (8 spec.). BL: 5.13-6.51; BW: 1.42-1.88; MAE: 0.9031.10; MBE: 0.623-0.793; OI: 132-145; PL: 1.34-1.85; PML: 1.27-1.62; PAW: 0.937-1.13; PW: 1.37-1.76; PI: 101-111; PWI: 146-161; EL: 3.32-4.29; EW: 

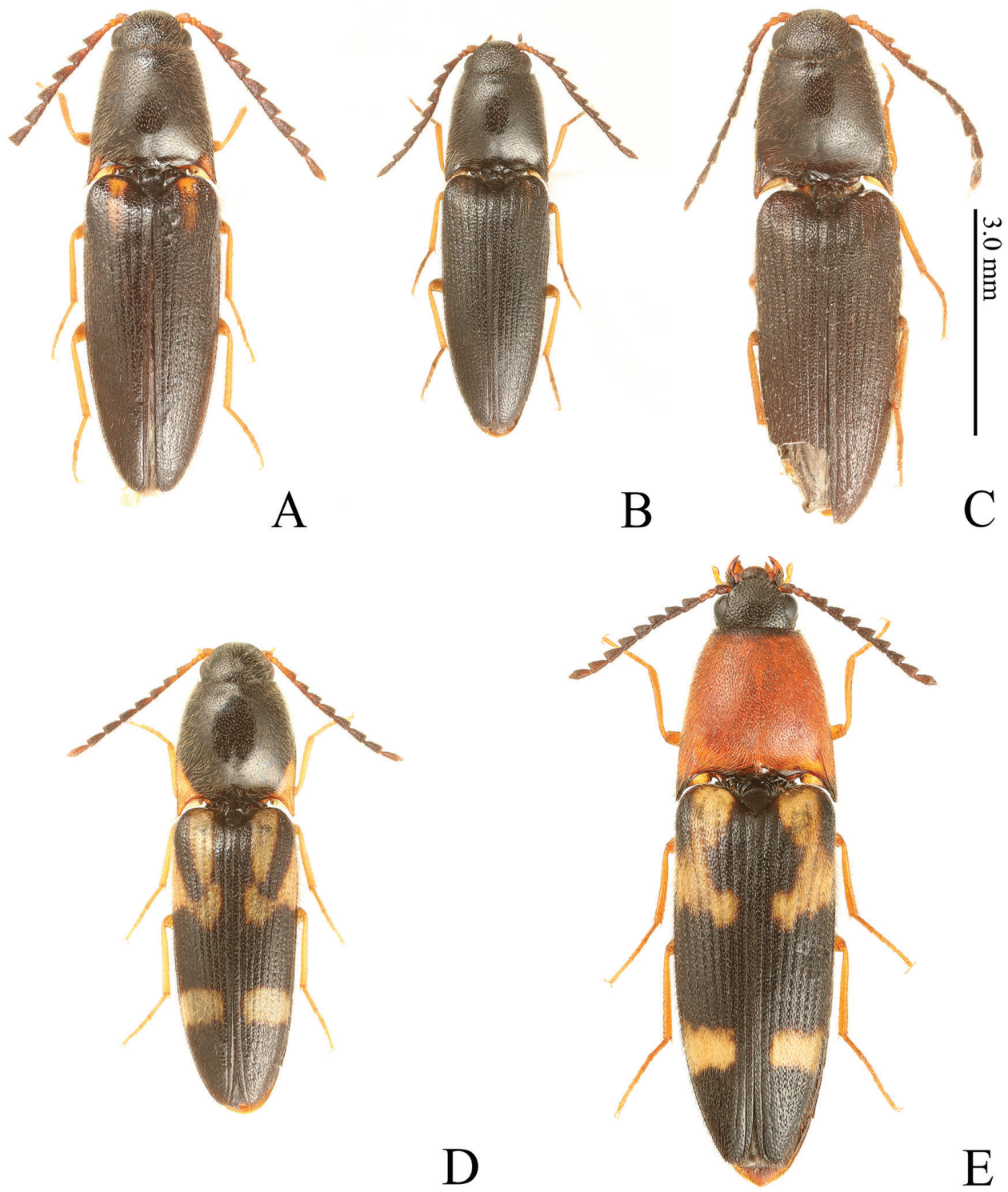

Figure 2. Gamepenthes spp., color variation A G. pictipennis, male [GP003] B G. pictipennis, male [GP012] C G. pictipennis, male, holotype of Nipponodrasterius alpicola D G. pictipennis, female [GP010] E G. versipellis, female [GV007].

1.42-1.88; EI: 222-238; BI: 212-247. Female (5 spec.). BL: 5.65-7.25; BW: 1.551.96; MAE: 0.915-1.14; MBE: 0.653-0.856; OI: 133-140; PL: 1.61-2.11; PAW: 0.943-1.17; PML: 1.40-1.87; PW: 1.47-1.91; PI: 108-112; PWI: 155-163; EL: 3.57-4.72; EW: 1.55-1.96; EI: 228-244; BI: 209-237. 


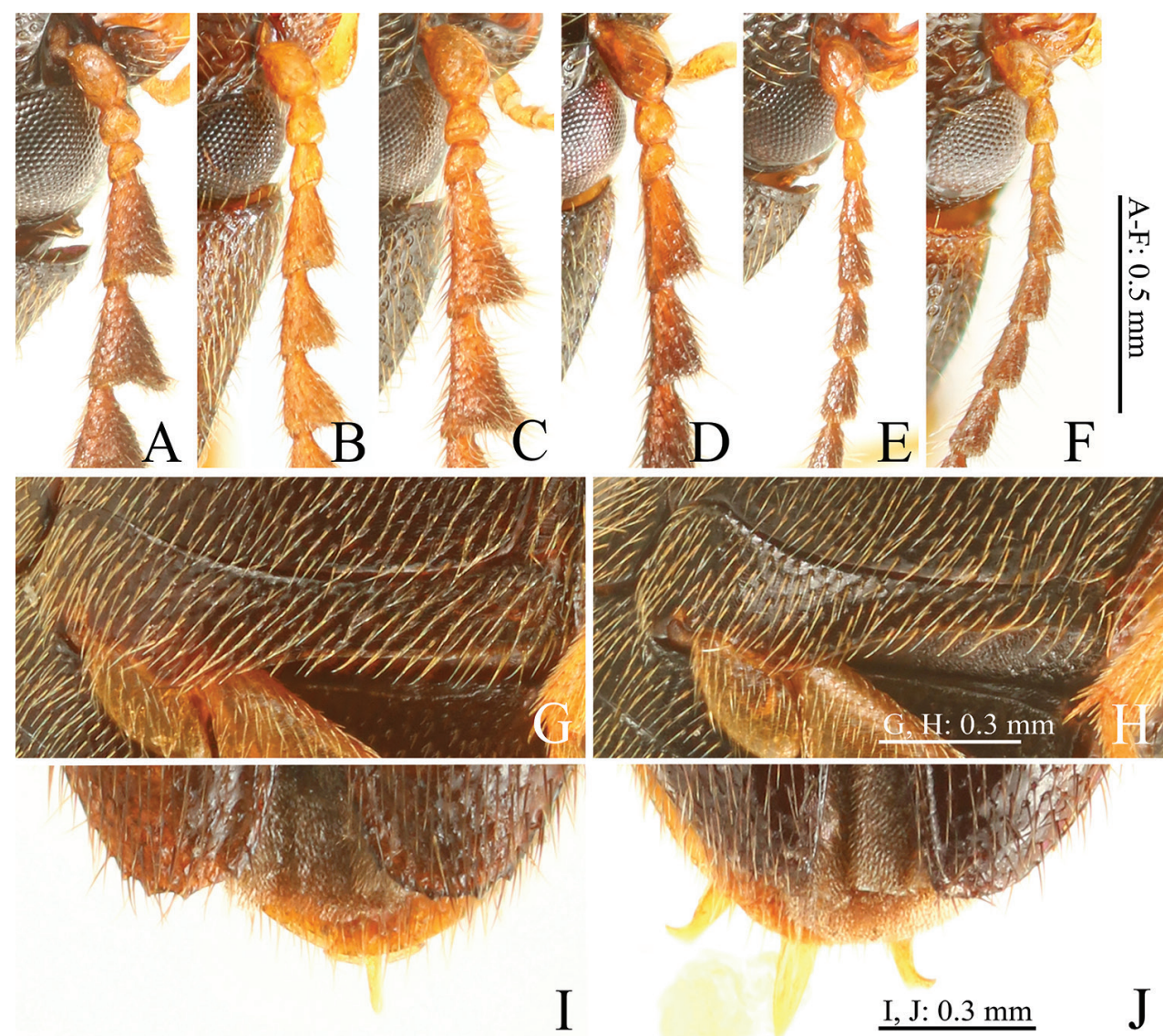

Figure 3. Gamepenthes spp. A-F right basal antennomeres $\mathbf{G}, \mathbf{H}$ left metacoxal plate $\mathbf{I}$, J apices of elytra A G. ornatus, male [GO003] B G. ornatus, female [GO009] C G. pictipennis, male [GP010] D G. pictipennis, female [GP011] E G. similis, male [GS003] F G. similis, female [GS006] G, I G. versipellis [GV011] H G. pictipennis [GP010] J G. pictipennis [GP003].

Distribution. Japan: Hokkaido, Honshu, Shikoku, Kyushu. Russia: Kunashir Island. The specimen examined from Hokkaido [GP001] is from the northern limit of the distribution of the genus.

\section{Gamepenthes similis (Lewis, 1894)}

Figures 1C, 3E, F, 7C

Melanoxanthus similis Lewis, 1894: 182 (original description; type locality: Fukushima

[Kisofukushima in Nagano Prefecture], Nikko in Tochigi Prefecture and Osaka in

Osaka Prefecture, Honshu, Japan).

Gamepenthes similis (Lewis, 1894); Kishii 1959: 59 (changed generic status). 
Note. Ôhira (1970a) transferred Melanoxanthus similis to the genus Gamepenthes, although Kishii (1959) had already suggested that the species belongs to Gamepenthes.

Material examined. 1 male, Japan, Honshu, Fukushima Prefecture, 31 VII 1989, K. Shindô leg. [GS001]; 1 male, 2 females, Japan, Honshu, Kyoto Prefecture, Kyoto City, Sakyô Ward, Hirogawara-obana Town, Sasari-tôge Pass, 698 m, 35.27546N, $135.72935^{\circ} \mathrm{E}, 1$ VIII 2020, Kôichi Arimoto leg., by beating shurbs [male: GS008; females: GS009, GS010]; 1 male, Japan, Honshu, Nara Prefecture, Tenkawa Village, Tsubonouchi Forest Road, 21 VII 2008, Hisayuki Arimoto leg. [GS002]; 2 males, Japan, Honshu, Wakayama Prefecture, Tanabe City, Mt. Gomadan-zan, 18 VII 2019, Hisayuki Arimoto leg. [GS003, 004]; 1 male, 1 female, Japan, Honshu, Tottori Prefecture, Saihaku District, Daisen Town, Mt. Dai-sen, 30 VII 2016, Akihiko Watanabe leg. [male: GS005; female: GS006]; 1 female, Japan, Honshu, Hiroshima Prefecture, Hikimi-tôge Pass, 7 VIII 1983, T. Itô leg. [GS007].

Diagnosis. Body robust (EI: 223-247, BI: 231-266). Prothorax black. Each elytron with yellow diagonal long marking basally. Antennomere II distinctly longer than wide; III longer than wide; IV robust (1.58-1.64 times longer than wide), shorter than II-III combined (0.67-0.76 times as long as II-III combined). Pronotum gradually widened posterad. Prosternal process weakly inclined dorsad $\left(23-37^{\circ}\right)$. Metacoxal plates not narrowed outwards, with outer edge wide. Apical edge of elytra rounded, without spines. Apex of parameres beyond apical-lateral hooks widely triangular (apex length 0.4 times width of parameres at expansion).

Measurements. Male (6 spec.) BL: 4.22-4.65; BW: 1.21-1.31; MAE: 0.7060.786; MBE: 0.487-0.576; OI: 136-145; PL: 1.11-1.25; PML: 0.924-1.07; PAW: 0.702-0.805; PW: 1.13-1.24; PI: 97.6-101; PWI: 154-165; EL: 2.84-3.21; EW: 1.21-1.31; EI: 234-247; BI: 246-266. Female (4 spec.). BL: 4.39-4.95; BW: 1.311.42; MAE: 0.728-0.819; MBE: 0.523-0.600; OI: 136-140; PL: 1.25-1.41; PML: 1.03-1.20; PAW: 0.736-0.832; PW: 1.20-1.37; PI: 103-105; PWI: 153-165; EL: 2.92-3.34; EW: 1.31-1.42; EI: 223-237; BI: 231-244.

Distribution. Japan: Honshu, Shikoku, Kyushu.

\section{Gamepenthes versipellis (Lewis, 1894)}

Figures 1D, 2E, 3G, 3I, 4C, D, 7D

Megapenthes versipellis Lewis, 1894: 47 (original description; type locality: Chiûzenji in Tochigi Prefecture and Kashiwagi in Nara Prefecture, Honshu, and Junsai, Sapporo and Otaru in Hokkaido, Japan).

Gamepenthes versipellis (Lewis, 1894); Kishii 1958: 29 (changed generic status).

Megapenthes versipellis aberrant form nigerrimus Nakane, 1958: 86 (original description; type locality: Mt. Osore-zan, Aomori Prefecture, Honshu, Japan).

Megapenthes versipellis aberrant form sexpunctatus Nakane, 1958: 86 (original description; type locality: Mt. Osore-zan, Aomori Prefecture, Honshu, Japan). 


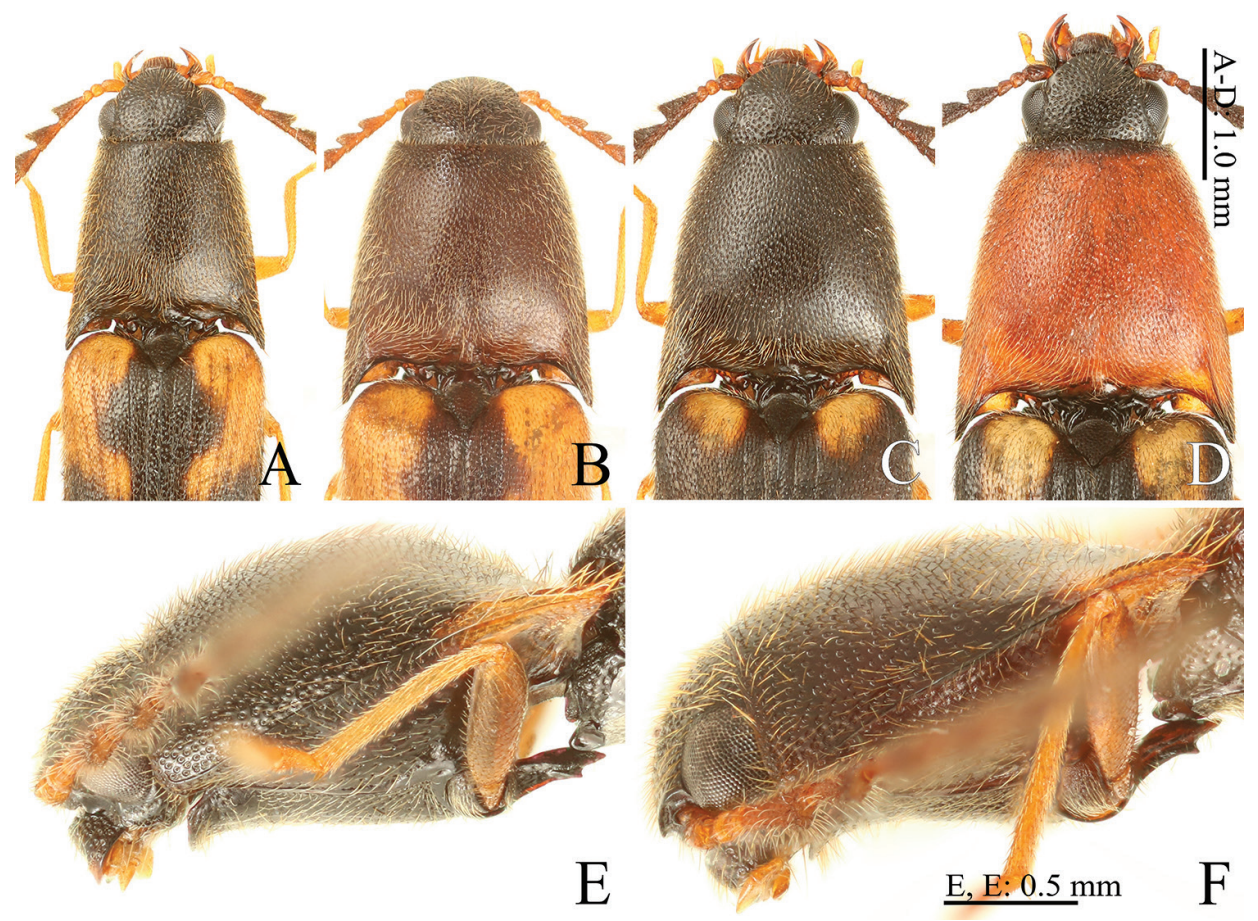

Figure 4. Gamepenthes spp., head and prothorax A G. ornatus, male [GO003] B G. ornatus, female [GO002] C G. versipellis, male [GV006] D G. versipellis, female [GV007] E G. pictipennis, male [GP010] F G. yoshidai, male [GY001] A-D dorsal view E, F lateral view.

Megapenthes versipellis aberrant form octopustularus Nakane, 1958: 87 (original description; type locality: Mt. Osore-zan, Aomori Prefecture, Honshu, Japan).

Megapenthes versipellis aberrant form interruptus Nakane, 1958: 87 (original description; type locality: Mt. Osore-zan, Aomori Prefecture, Honshu, Japan).

Megapenthes versipellis var. shirozui Kishii, 1958: 31 (original description; type locality: Mt. Kujyû-san, Ôita Prefecture, Kyushu, Japan).

Note. The species exhibits elytral color variation. Based on this, Nakane (1958) and Kishii (1958) described four forms and one variant.

Material examined. 1 female, Japan, Hokkaido, Samani District, Samani Town, Mt. Apoi-dake, 9 VIII, 1974, H. Miyauchi leg. [GV001]; 1 male, Japan, Honshu, Nagano Prefecture, Mt. Kisokomaga-take, 31 VII 1976, Ryôji Toyoshima leg. [GV002]; 1 male, Japan, Honshu, Nagano Prefecture, Mt. Yatsuga-take, 26 VII 1979, Katsuhiko Kitagawa leg. [GV003]; 1 male, Japan, Honshu, Gifu Prefecture, Ôno District, Shôkawa Village, Ogamigô, 12 VIII 1995, N. Yuzawa leg. [GV004]; 1 male, Japan, Honshu, Nara Prefecture, Yoshino District, Kamikitayama Village, Mt. Ôdaigahara, 14 VII 1985, Hisayuki Arimoto leg. [GV005]; 1 male, 3 females, Japan, Honshu, Nara Prefecture, Yoshino District, Totsukawa Village, Asahi, Mt. Syakaga-take, 1450 m, 


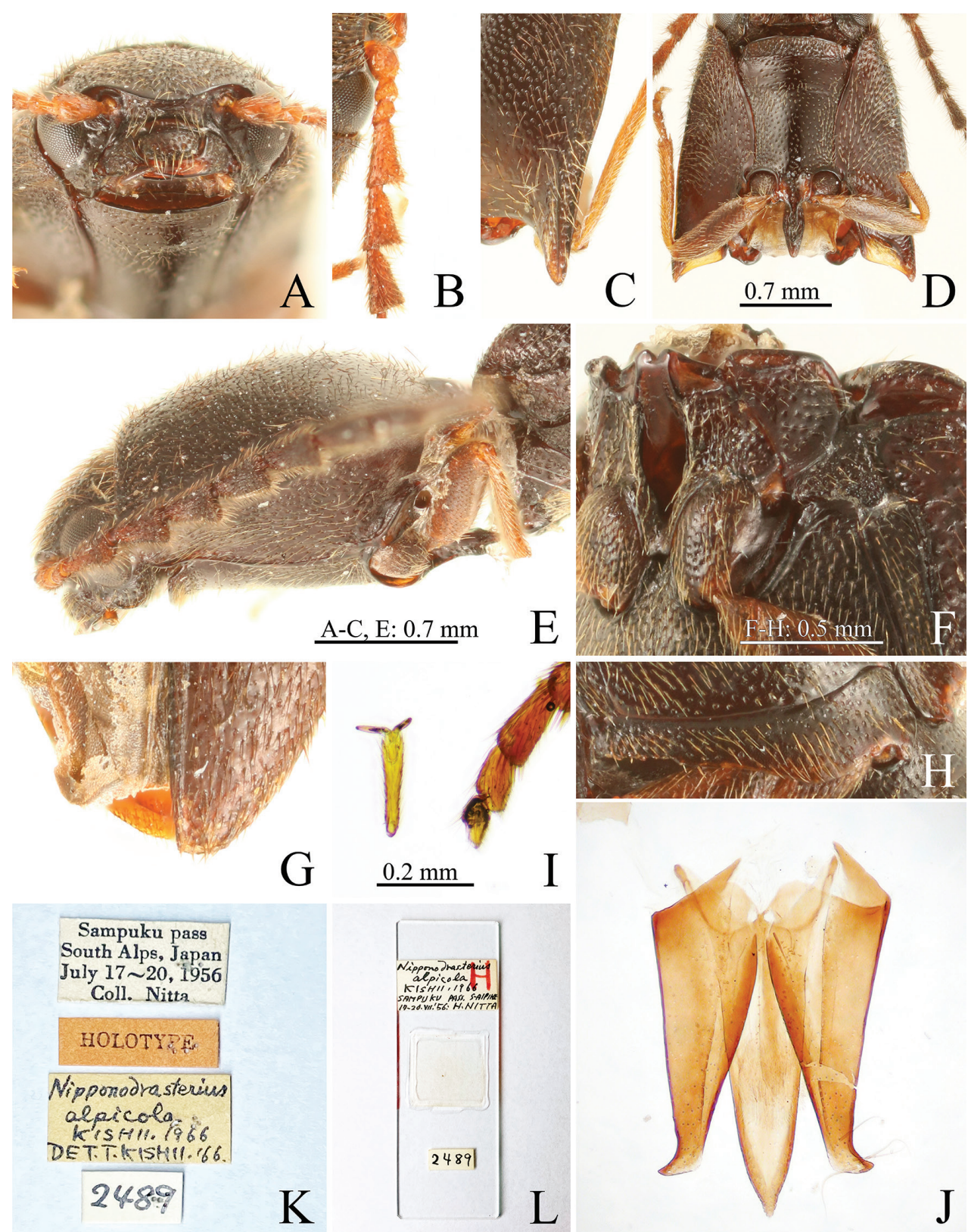

Figure 5. Holotype of Nipponodrasterius alpicola $\mathbf{A}$ head, anterior view $\mathbf{B}$ right basal antennomeres $\mathbf{C}$ hind angles of pronotum $\mathbf{D}$ prothorax, ventral view $\mathbf{E}$ prothorax, lateral view $\mathbf{F}$ mesosternum $\mathbf{G}$ apices of elytra $\mathbf{H}$ right metacoxal plate $\mathbf{I}$ left mid tarsus and claw $\mathbf{J}$ aedeagus $\mathbf{K}$ labels $\mathbf{L}$ aedeagus slide.

4 VIII 2019, Hisayuki Arimoto leg., from flowers of Tilia japonica [male: GV006; females: GV007-GV009]; 2 males, Japan, Honshu, Nara Prefecture, Kamikitayama Village, Amagase, 11 VII 2004, Hisayuki Arimoto leg. [GV010, GV011]; 1 male, 
Japan, Honshu, Nara Prefecture, Tenkawa Village, 23 VII 1989, H. Nomura leg. [GV012]; 1 female, Japan, Honshu, Hyôgo Prefecture, Shisô City, Haga Town, Tokura, Mt. Hyôno-sen, 1 VII 2015, S. Sugimoto leg. [GV013]; 1 female, Japan, Shikoku, Ehime Prefecture, Mt. Ishizuchi-san, 26 VII 1979, Kiyoshi Matsuda leg. [GV014]; 1 male, 2 females, Japan, Kyushu, Miyazaki Prefecture, Nishiusuki District, Gokase Town, Mt. Shiraiwa-yama, 1347 m, 32³4'13.7"N, 13106'51.6"E, 30 VII 2011, Kôichi Arimoto leg. [male: GV015; females: GV016, GV017].

Diagnosis. Body robust (EI: 226-243, BI: 206-222). Prothorax black in male; female with pronotum and hypomeron red, prosternum black. Each elytron with pale orange U-shaped marking basally; basal marking partly reduced in some. Antennomere II shorter than wide in male, slightly longer than wide in female; III shorter than wide in male, longer than wide in female; IV robust (1.48-1.72 times longer than wide in male, 1.66-1.75 times longer than wide in female), longer than II-III combined (1.72-1.99 times as long as II-III combined in male, 1.08-1.14 times as long as II-III combined in female). Prosternal process weakly inclined dorsad $\left(17-26^{\circ}\right)$. Metacoxal plates not narrowed outwards, with outer edge wide. Apical edge of elytra serrate. Apex of parameres beyond pre-apical expansions widely triangular (apex length 0.5-0.6 times width of parameres at expansion).

Measurements. Male (8 spec.). BL: 6.60-7.53; BW: 1.92-2.19; MAE: 1.07-1.19; MBE: 0.709-0.831; OI: 141-154; PL: 1.98-2.33; PML: 1.73-1.96; PAW: 1.101.22; PW: 1.92-2.19; PI: 103-110; PWI: 172-184 ; EL: 4.30-5.03; EW: 1.88-2.11; EI: 229-243; BI: 206-222. Female (9 spec.). BL: 7.08-9.24; BW: 2.03-2.60; MAE: 1.12-1.30; MBE: 0.753-0.907; OI: 142-149; PL: 2.20-2.77; PML: 1.89-2.38; PAW: 1.15-1.39; PW: 2.03-2.60; PI: 106-112; PWI: 176-191; EL: 4.64-6.04; EW: 2.02-2.58; EI: 226-241; BI: 207-219.

Distribution. Japan: Hokkaido, Honshu, Shikoku, Kyushu. Russia: Kunashir Island. This species was also recorded from China and Oriental region (Cate 2007). Schimmel (2003, 2004, 2006) and Schimmel and Tarnawski (2009) recognized 18 species from China and Indochina: however, G. versipellis had not been confirmed from the area. Therefore, the records of G. versipellis from China and the Oriental region were probably based on misidentifications of allied species, such as $G$. hubeiensis Schimmel, 2003, G. holzschuhi Schimmel, 2003, G. kresli Schimmel \& Tawnawski, 2009, G. sausai Schimmel, 2003 and G. sichuanensis Schimmel, 2003.

\section{Gamepenthes yoshidai Ôhira, 1995}

Figures 1E, 4F, 7E

Gamepenthes yoshidai Ôhira, 1995b: 27 (original description; type locality: Mt. Tsurugisan, Tokushima Prefecture, Shikoku, Japan).

Material examined. Paratype. 1 female, Japan, Shikoku, Tokushima Prefecture, Miyoshi City, Mt. Tsurugi-san, Minokoshi, 31 VII 1982, Yûji Kurota leg. 

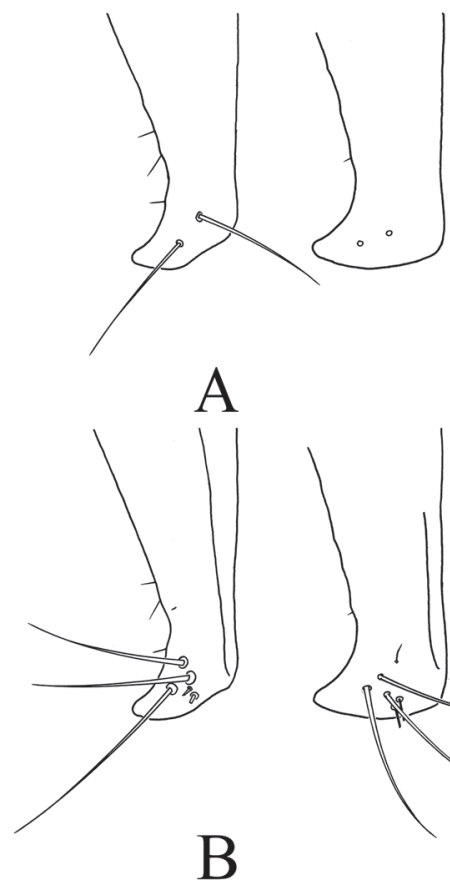
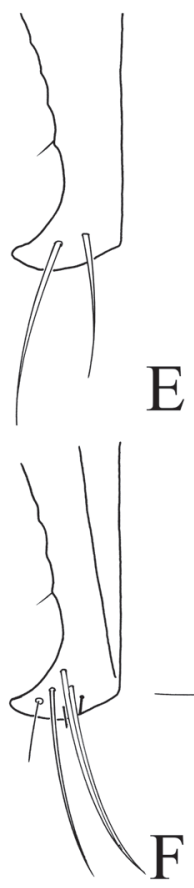
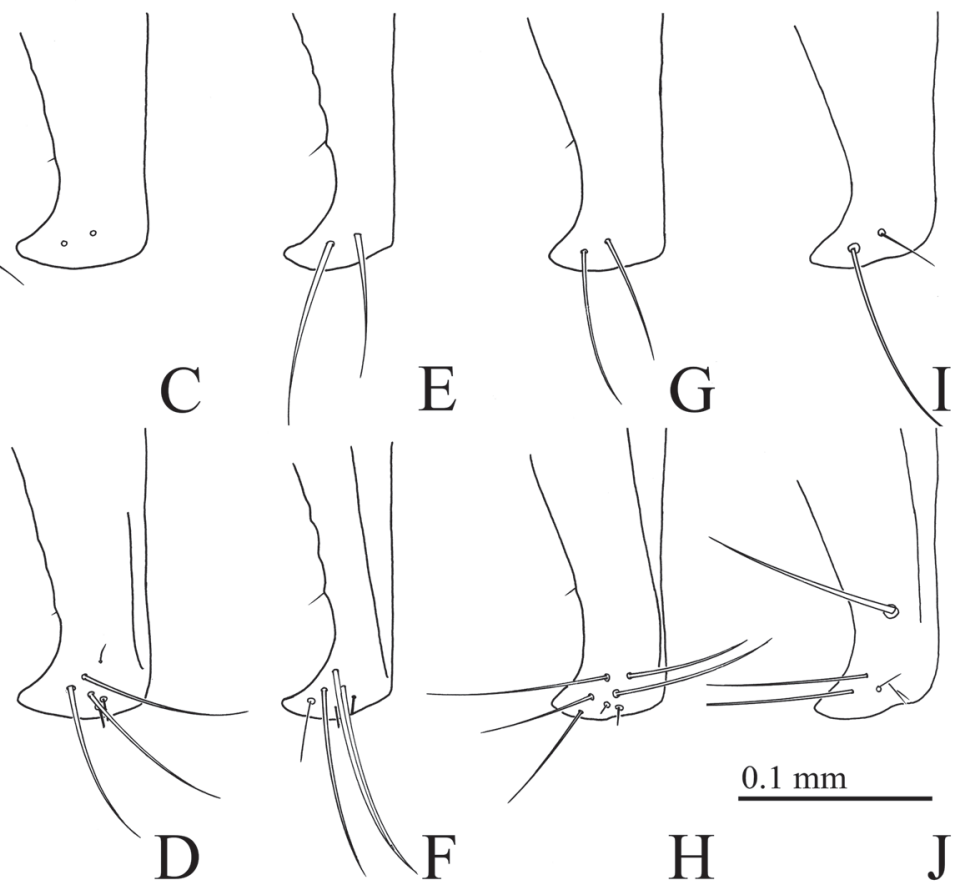

Figure 6. Gamepenthes pictipennis, male, apex of left paramere of aedeagus A, B [GP006] C, D [GP007] E, F [GP009] G, H [GP012] I, J holotype of Nipponodrasterius alpicola A, C, E, G, I dorsal side B, D, F, H, J ventral side.

Non-type material. 2 males, Japan, Shikoku, Tokushima Prefecture, Naka District, Naka Town (formerly Kisawa Village), Mt. Takashiro-yama, 31 VII 1993, Mitsuo Kakô leg. [GY001, GY002].

Diagnosis. Body slender (EI: 259-265, BI: 254-282). Prothorax black; hind angles of pronotum yellow. Each elytron with three separate yellow markings basally. Antennomere II shorter than wide in male, almost as long as wide in female; III shorter than wide in male, longer than wide in female; IV elongate (1.92-2.06 times longer than wide in male, 2.17 times longer than wide in female), longer than II-III combined (1.27-1.77 times as long as II-III combined). Prosternal process strongly inclined dorsad $\left(43-50^{\circ}\right)$. Metacoxal plates narrowed outwards on lateral half, with outer edge narrow. Apical edge of elytra rounded, without spines. Apex of parameres beyond pre-apical expansions widely triangular (apex length $0.3-0.4$ times width of parameres at expansion).

Measurements. Male (2 spec.). BL: 6.96-7.29; BW: 1..87-1.98; MAE: 1.171.21; MBE: 0.711-0.752; OI: 161-164; PL: 1.72-1.87; PML: 1.51-1.65; PAW: 1.17-1.23; PW: 1.74-1.87; PI: 98.8-100; PWI: 148-152; EL: 4.85-5.13; EW: 1.871.98; EI: 259; BI: 275-282. Female (1 spec.). BL: 7.20; BW: 1.89; MAE: 1.15; MBE: 0.743; OI: 154; PL: 1.98; PML: 1.71; PAW: 1.16; PW: 1.82; PI: 109; PWI: 157; EL: 5.02; EW: 1.89; EI: 265; BI: 254.

Distribution. Japan: Shikoku. This species may be endemic to Shikoku, Japan. 

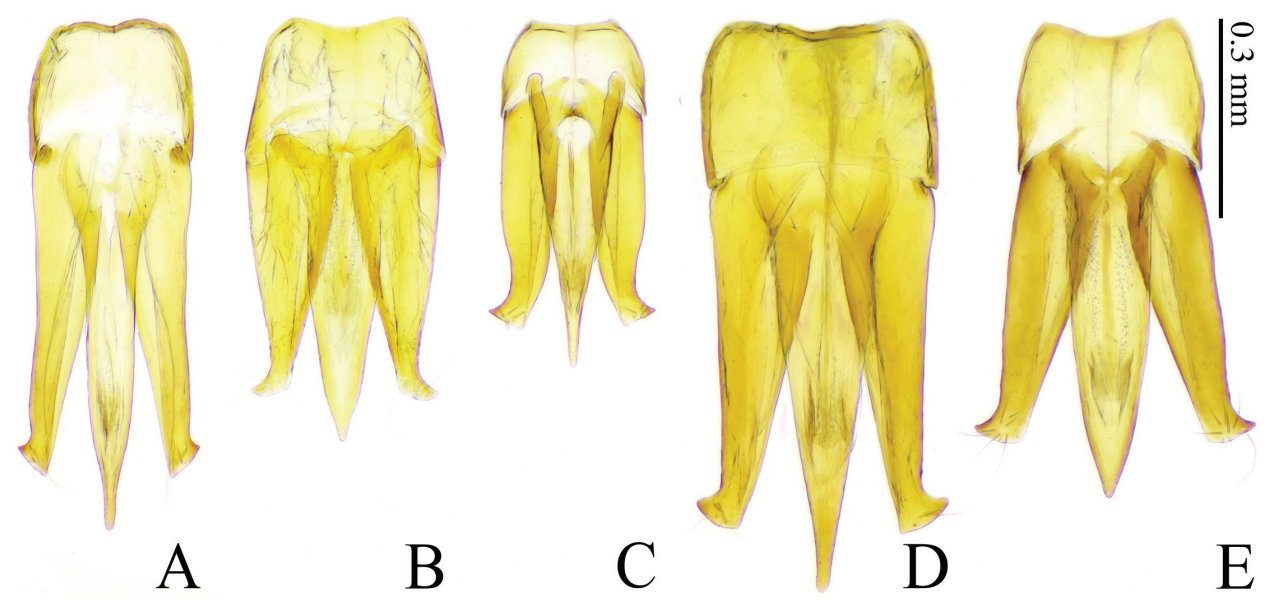

Figure 7. Gamepenthes spp., male, aedeagus, ventral side A G. ornatus [GO006] B G. pictipennis [GP006] C G. similis [GS008] D G. versipellis [GV003] E G. yoshidai [GY002].

\section{Discussion}

The holotype of $N$. alpicola has an oval head capsule in lateral view (Fig. 5E), inferior mouth-parts (Fig. 5E), mesocoxal cavity open to the mesepimeron and mesepisternum (Fig. 5F), mesosternum separated by suture from metasternum (Fig. 5F), and claws simple and without basal setae (Fig. 5I). These features are diagnostic for members of the subfamily Elaterinae (Stibick 1979). Moreover, the holotype has the following key features for the genus Gamepenthes of the tribe Megapenthini (Ôhira 1970a; Schimmel 2003): supra-antennal carina compete and depressed medially (Fig. 5A), vertical distance between supra-antennal carina and labrum narrowed medially in anterior view (Fig. 5A), antennomeres II and III short (Fig. 5B), antennomeres IV-X serrate (Fig. $5 \mathrm{~B}$ ), hind angles of pronotum unicarinate (Fig. $5 \mathrm{C}$ ), procoxal cavity partly closed by a projection of the hypomeron, prosternal process concave between procoxae (Fig. 5D) and with subapical tooth (Fig. 5E), and elytral surface with small tubercle-like prominences on anterior edge of punctures (Fig. 2C) (the elytral surface clearly granulated in Kishii 1966). Kishii (1987) stated that tarsomere IV of $N$. alpicola was slightly enlarged apically, but the tarsomere IV of the holotype is simple, which matches the diagnosis of Gamepenthes and Megapenthini (Fig. 5I). Therefore, we concluded that Nipponodrasterius should be a junior synonym of Gamepenthes.

We found a slide of the male genitalia labeled as belonging to the holotype of $N$. alpicola (matching specimen number and label information) in OMNH (Fig. 5K, L), although the sex and genitalia of the holotype were not mentioned in the original description. We found that $N$. alpicola should be a junior synonym of G. pictipennis by comparing the aedeagi of the Japanese Gamepenthes species (Figs 5J, 6, 7) and their aedeagus shown in Ôhira (1970b, 1995a). Gamepenthes pictipennis is also distinguished from its congeners by a combination of body size, antennae, prosternal pro- 
cess, metacoxal plates, and apical edge of the elytra (see above key and diagnoses). The holotype of $N$. alpicola shares these features (Fig. 5). We also found that G. pictipennis has variable pronotum and elytral coloration, ranging from a black base with clear yellow markings (Figs 1B, 2D) to having fewer yellow markings (Fig. 2A) to entirely black (Fig. 2B). Even the black specimens show slight yellow markings, and the hind angles of the pronotum and anterior parts of that elytra are slightly yellow tinged (Fig. 2B), as in the holotype (Figs 2C, 5C). We conclude the holotype of $N$. alpicola is a melanic specimen of $G$. pictipennis.

\section{Acknowledgments}

We thank Shigehiko Shiyake (OMNH) for providing access to the facilities and material, as well as Yûji Kurota (Tokushima, Japan), Ryô Noda (Fukuoka, Japan), Yûji Tsutsumiuchi (Ôita, Japan) and Akihiko Watanabe (Okayama, Japan) for providing materials. We are also grateful to Wataru Suzuki (Tokyo, Japan) for his sincere cooperation in finding the holotype of $N$. alpicola.

\section{References}

Arimoto K, Suzuki W (2020) First record and two new species of the genus Limonius Eschscholtz (Coleoptera, Elateridae, Dendrometrinae) from Taiwan. Zootaxa 4742(1): 89-104. https://doi.org/10.11646/zootaxa.4742.1.5

Bouchard P, Bousquet Y, Davies AE, Alonso-Zarazaga MA, Lawrence JF, Lyal CHC, Newton AF, Reid CAM, Schmitt M, Ślipiński SA, Smith ABT (2011) Family-group names in Coleoptera (Insecta). Zookeys 88: 1-972. https://doi.org/10.3897/zookeys.88.807

Candèze ECA (1857) Monographie des Élatérides. Tome premier. Mémoires de la Société Royale des Sciences de Liège 12: 1-400. https://doi.org/10.5962/bhl.title.8958

Cate PC (2007) Family Elateridae. In: Löbl I, Smetana A (Eds) Catalogue of Palaearctic Coleoptera, Vol. 4. Elateroidea - Derodontoidea - Bostrichoidea - Lymexyloidea - Cleroidea - Cucujoidea. Apollo Books, Stenstrup, 94-209.

Costa C, Lawrence JF, Rosa SP (2010) Elateridae Leach, 1815. In: Leschen RAB, Beutel RG, Lawrence JF (Eds) Coleoptera, Beetles. Volume 2. Morphology and Systematics (Elateroidea, Bostrichiformia, Cucujiformia partim). In: Kristensen NP, Beutel RG (Eds) Handbook of Zoology, Arthropoda: Insecta. Walter de Gruyter GmbH \& Co. KG, Berlin/New York, 75-103.https://doi.org/10.1515/9783110911213.75

Douglas H (2011) Phylogenetic relationships of Elateridae inferred from adult morphology, with special reference to the position of Cardiophorinae. Zootaxa 2900: 1-45. https://doi. org/10.11646/zootaxa.2900.1.1

Fleutiaux E (1919) Insectes Coléoptères 13. Elateridae, Trixagidae et Melasidae. In: Voyage de Ch. Alluaud et R. Jeannel en Afrique Orientale (1911-1912) Résultats Scientifiques. Lhomme, Paris, 119 pp. 
Fleutiaux E (1928) Les Élatérides de l' Indochine Francaise (Catalogu raisonné). Deuxième Partie. Encylclopédie Entomologique, Coléoptèra 3: 103-177.

Gistel JNFX (1848) Faunula monacensis cantharologica. Isis von Oken 1848(7): 4-6 (unnumbered).

Gurjeva EJ (1973) Novaja Triba zhukov-shchelkunov Megapenthini Tribus n. (Col., Elateridae). Zoologischeskii Zhurnal 52: 448-451. [In Russian]

Hayek CMF von (1973) A reclassification of the subfamily Agrypninae (Coleoptera: Elateridae). Bulletin of the British Museum (Natural History) Entomology, Supplement 20: 1-309.

Kishii T (1958) Snappers from Kyûshû-District, Japan, collected by Prof. Takashi Shirôzu. The Entomological Review of Japan 9(1): 27-32. [In Japanese, with English title and description] Kishii T (1959) Elateridae of Amami-Oshima collected by Prof. Shirozui. Akitu 8(3): 57-64, pls. I-II.

Kishii T (1966) Some new forms of Elateridae in Japan (IV). Bulletin of Heian High School 10: 1-11, pls. I-II.

Kishii T (1968) Some new forms of Elateridae in Japan (V). Bulletin of Heian High School 13: $1-15$, pls. I-III.

Kishii T (1987) A taxonomic study of the Japanese Elateridae (Coleoptera), with the keys to the subfamilies, tribes and genera. T. Kishii, Kyoto, 262pp.

Kishii T (1999) A check-list of the family Elateridae from Japan (Coleoptera). Bulletin of the Heian High School 42: 1-144.

Kundrata R, Kubaczkova M, Prosvirov AS, Douglas HB, Fojtikova A, Costa C, Bousquet Y, Alonso-Zarazaga MA, Bouchard P (2019) World catalogue of the genus-group names in Elateridae (Insecta, Coleoptera). Part I: Agrypninae, Campyloxeninae, Hemiopinae, Lissominae, Oestodinae, Parablacinae, Physodactylinae, Pityobiinae, Subprotelaterinae, Tetralobinae. Zookeys 839: 83-154. https://doi.org/10.3897/zookeys.839.33279

Leach WE (1815) Entomology. In: Brewster D (Ed.) The Edinburgh Encyclopedia. 9. William Blackwood, Edinburgh: 57-172.

Lewis G (1894) On the Elateridae of Japan. The Annals and Magazine of Natural History (series 6) 13: 26-48, 182-201. https://doi.org/10.1080/00222939408677683

Nakane T (1958) On the Coleoptera of Shimokita Peninsula, northern end of Honshu, Japan (Insects I). Miscellaneous Reports of the Research Institute for Natural Resources 46/47: 83-92. [In Japanese, with English title and descriptions]

Ôhira H (1970a) Notes on some elaterid-beetles from Japan (Coleoptera) (V). The Entomological Review of Japan 22(2): 75-88. [pls. 8-11]

Ôhira H (1970b) Elateridae in Japan (VII). The Nature and Insects 5(10): 19-24. [In Japanese]

Ôhira H (1994) Notes on the genera and species of Conoderinae (Coleoptera: Elateridae) from Japan. Transactions of Essa entomological Society 2: 217-234. [In Japanese, with English description]

Ôhira H (1995a) On the Gamepenthes-species from Japan (Coleoptera: Elateridae). GekkanMushi 290: 14-17. [In Japanese, with English title]

Ôhira H (1995b) New or little-known Elateridae (Coleoptera) from Japan, XXXII. Elytra 23(1): 25-30. 
Ootsuka et al. (1981) Notes on elaterid beetles in Kumamoto Prefecture (3). Transactions of the Kumamoto Entomological Society 26(2): 1-14. [In Japanese]

Prosvirov AS (2013) To the knowledge of the fauna of click-beetles (Coleoptera: Elateridae) of the Siberia and Far East of Russia. Russian Entomological Journal 22(4): 275-282.

Schimmel R (2003) Die Megapenthini-Arten Süd- und Südostasiens. Zweiter Teil: Acumenator, Anchastelater, Dicarinator, Gamepenthes, Ganoxanthus, Martiniana und Simodactylus. Pollichia-Buch 42: 1-261. [In German]

Schimmel R (2004) Die Megapenthini-Arten Süd- und Südostasiens. Dritter Teil: Abelatter, Amamipenthes, Cateanus n. gen., Friedrichiellus n. gen., Hayekpenthes, Megapenthes, Melanoxanthus, Pengamethes, Platianeus n. gen., Sawadapenthes und Wallaceus n. gen. PollichiaBuch 45: 1-504. [In German]

Schimmel R (2006) Neue Megapenthini-, Physorhinini-, Diminae- und Senodoniina-Arten aus Südostasien (Insecta: Coleoptera, Elateridae). Mitteilungen der Pollichia 92: 107-130. [In German]

Schimmel R, Tarnawski D (2009) New species of the tribe Megapenthini Gurjeva, 1973 (Coleoptera, Elateridae) from Asia. Annales Zoologici 59(4): 629-639. https://doi. org/10.3161/000345409X484991

Schwarz O (1898) Beschreibung neuer Elateriden. Deutsche Entomologische Zeitschrift 1898 (1): 129-156. https://doi.org/10.1002/mmnd.48018980117

Stibick JNL (1979) Classification of the Elateridae (Coleoptera). Relationships and classification of the subfamilies and tribes. Pacific Insects 20(2-3): 145-186.

Thomson CG (1859) Skandinaviens Coleoptera, synoptiskt bearbetade. Tom. I. Berlingska Boktryckerit, Lund, 290 pp. https://doi.org/10.5962/bhl.title.138677 